\title{
PHYSICAL STUDY OF A BRIGHT RIM \\ IN THE HORSEHEAD NEBULA
}

\author{
R. LOUISE and C. SAPIN
}

Observatoire de Marseille, 2, place Le Verrier, F 13004 Marseille, France

\begin{abstract}
The wellknown 'Horsehead' nebula is photographed with narrow interference filters centered respectively on $\mathrm{H} \alpha$ and $[\mathrm{N} \mathrm{II]}(\lambda=6584 \AA)$. The [N II]/H $\alpha$ ratio is deduced and its variation towards the Horsehead bright rim is studied. It is shown that the maximum value of the temperature is not reached on the rim itself but in a narrow zone located just behind it and ahead of the dark matter.
\end{abstract}

\section{Introduction}

Among the most intriguing features in $\mathrm{H}$ II regions are 'elephant trunk' structures, so designated because of their elongated form; they also are called bright rims (Pottasch, 1958). They are characterised by neutral dark matter intruding into the $\mathrm{H}$ II zone and are brighter than the rest of the nebula. An important theoretical work done by Pottasch shows that both electron density and temperature increase towards the bright rims. Up to now, only a few observations (Osterbrock, 1957; Louise, 1970) have been made concerning them.

Our purpose is to measure the variation of temperature from the normal $\mathrm{H}$ II zone towards the bright rim by observing the ratio of $[\mathrm{N} \mathrm{II}(\lambda=6584 \AA)$ and $\mathrm{H} \alpha(\lambda=6563$ $\AA)$ emission lines. The wellknown and typical bright rim in IC 434, the Horsehead nebula, is chosen to be observed at the $120 \mathrm{~cm}$ telescope of the Haute Provence Observatory.

\section{Observational Method}

We may choose one of the observational methods described by Courtès et al. (1969). Unfortunately, the bright rims are generally small structures and high angular resolution observations appear to be the desirable.

The method we have adopted is based on the fact that if the $[\mathrm{N} \mathrm{II}] / \mathrm{H} \alpha$ ratio is known in one point (A) of the nebula it is possible to derive that ratio is at any point (B) by photographing the nebula through narrow interference filters $(\Delta \lambda=10 \AA)$ centered respectively on $\lambda=6584 \AA$ and $\mathrm{H} \alpha$.

Indeed we have:

$$
\frac{([\mathrm{N} \mathrm{II}] / \mathrm{H} \alpha)_{B}}{([\mathrm{~N} \mathrm{II}] / \mathrm{H} \alpha)_{\mathrm{A}}}=\frac{([\mathrm{N} \mathrm{II}])_{B}}{([\mathrm{~N} \mathrm{II}])_{\mathrm{A}}} \cdot \frac{(\mathrm{H} \alpha)_{\mathrm{A}}}{(\mathrm{H} \alpha)_{\mathrm{B}}} .
$$

The above formula shows that the $\mathrm{H} \alpha$ and [N II] plates may be taken separately with an adequate exposure time. Furthermore it may be interpreted as the $[\mathrm{N} \mathrm{II}] / \mathrm{H} \alpha$ relative variation over the whole nebula compared to the $[\mathrm{N} \mathrm{II}] / \mathrm{H} \alpha$ of the point $(A)$. 

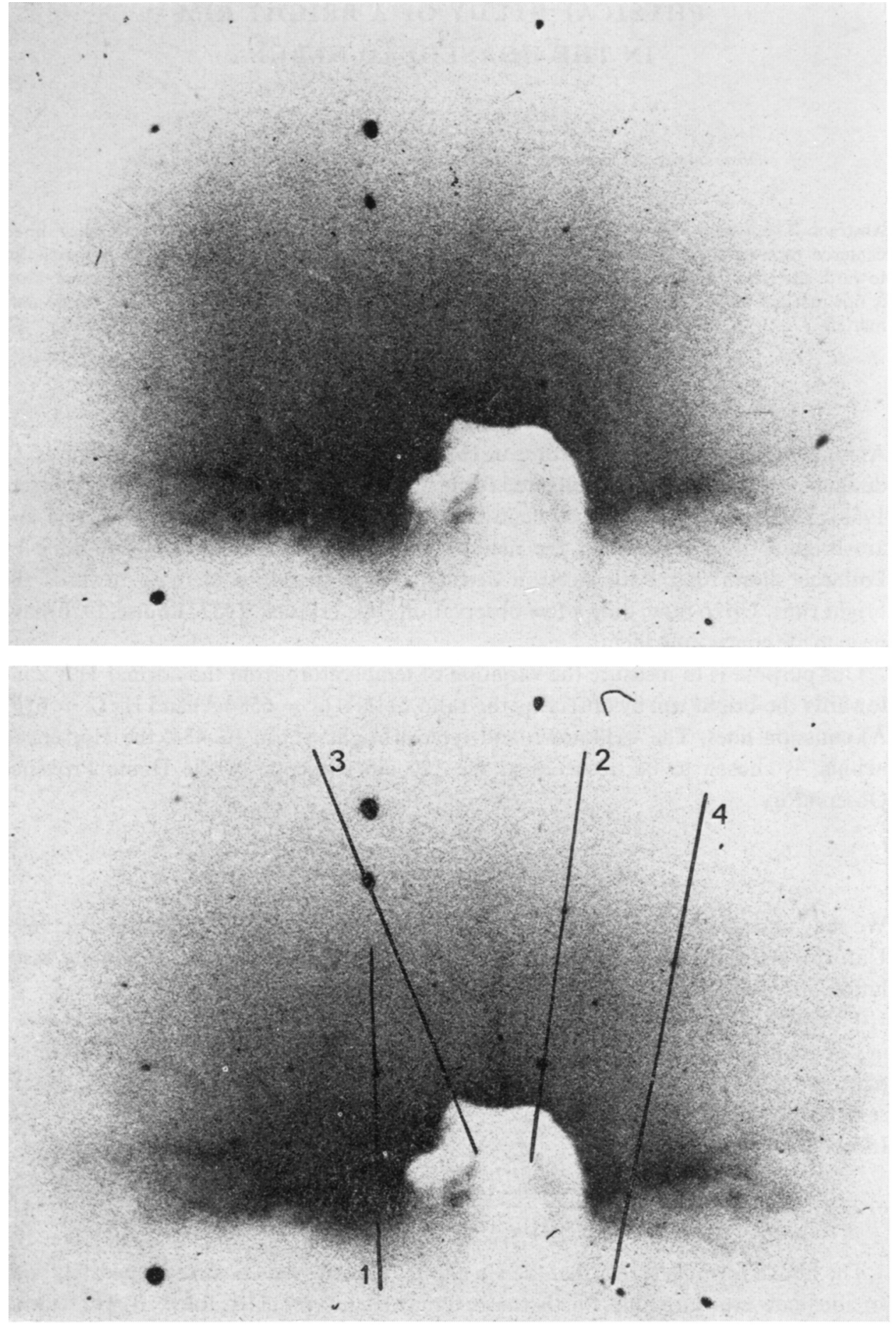

Fig. 1. Monochromatic exposures of the Horsehead nebula in $\mathrm{H} \alpha$ (top) and in [N II] (bottom). Lines show the four microphotometer scans. 
In this work the point $(\mathrm{A})$ is chosen at the top of the bright rim photometric profile. On the other hand, it is not necessary to know the $[\mathrm{N} \mathrm{II}] / \mathrm{H} \alpha$ of the point (A) if we only want to study the variation of that ratio from the normal $\mathrm{H}$ il zone towards the bright rim.

\section{Observation and Reduction}

Plates with various exposure times were taken with the classical Focal Reducer (Courtès, 1960), without Fabry-Perot etalon, at the Newtonian focus of the $120 \mathrm{~cm}$ telescope of the Haute Provence Observatory. Figure 1 shows the image of the Horsehead nebula taken in $\mathrm{H} \alpha$ and [N II] monochromatic light respectively. The exposure times, $40 \mathrm{~min}$ for $\mathrm{H} \alpha$ and $1^{\mathrm{h}} 30$ for [N $\mathrm{II}$ ], are adopted because they allow to obtain practically the same optical density on the Kodak 103a-E plate. The Joyce and Loebl microphotometer is used to record the brightness along 4 directions as indicated by Figure 1. These directions are adopted in a rather arbitrary way owing to the positions

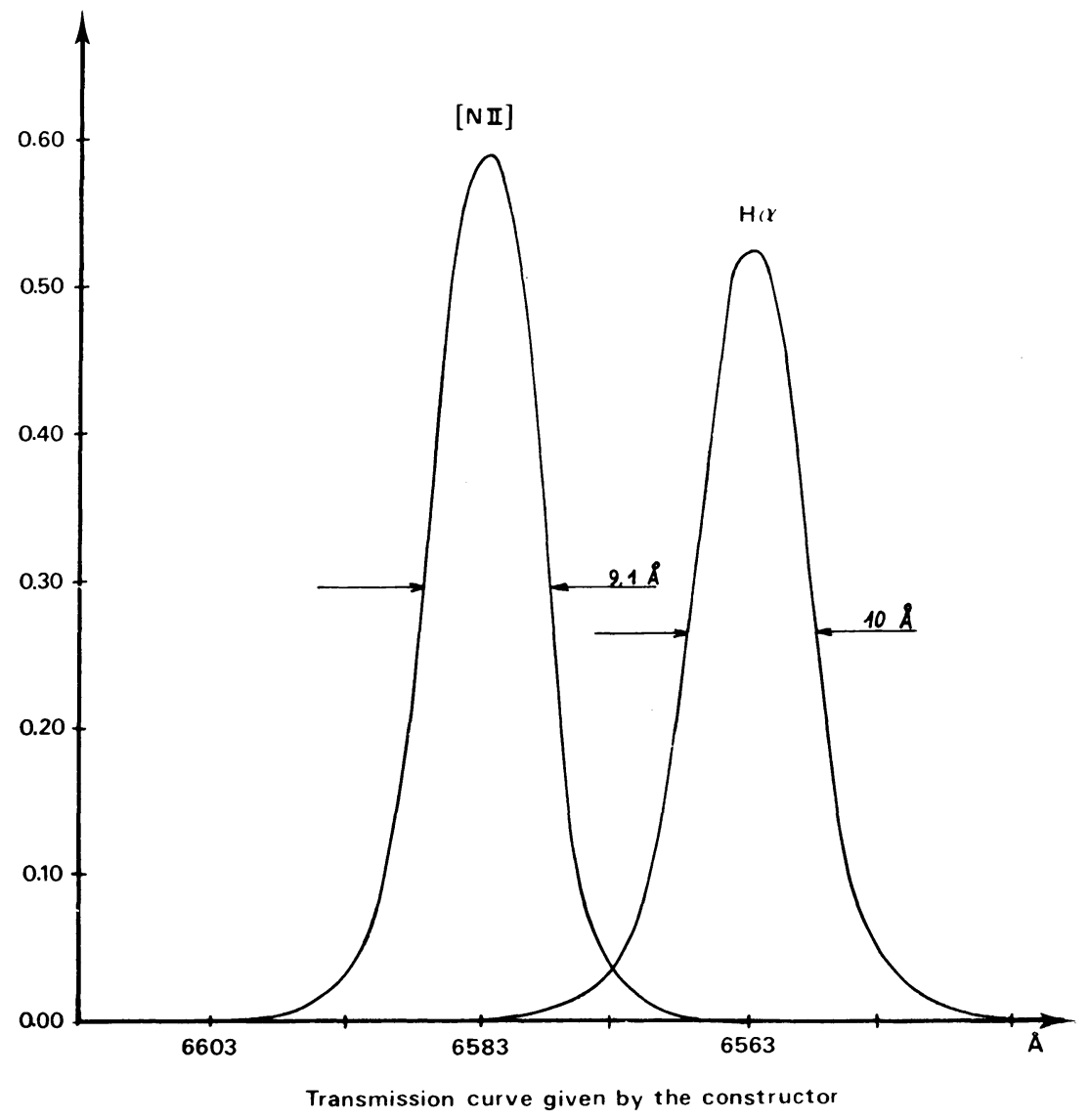

Fig. 2. Transmission curves of the two filters. 
of stars on the plate; but they must be as perpendicular as possible to the bright rim.

The photographic calibration is obtained by using the classical ETA photometric spectrograph of the Haute Provence Observatory.

The interference filters we used present a narrow bandwidth. A sample of their transmission curve is given in Figure 2. The $\mathrm{H} \alpha$ image is not in practice contaminated by [N II] light and vice versa. Although the bandwidth is narrow $(\Delta \lambda=10 \AA)$ and the continuum emission spectrum of the nebula is weak, the observed intensity is always overestimated.

Indeed, we have:

$$
\begin{aligned}
& I_{\mathrm{H} \alpha}(\text { observed })=I_{\mathrm{H} \alpha}(\text { Line })+\varepsilon(\text { continuum }) \\
& I_{[\mathrm{N} \mathrm{II}}(\text { observed })=I_{[\mathrm{N} \mathrm{II}}(\text { Line })+\varepsilon^{\prime}(\text { continuum }) .
\end{aligned}
$$

$\varepsilon$ and $\varepsilon^{\prime}$ are respectively the continuum intensity of the nebula admitted by $\mathrm{H} \alpha$ and $[\mathrm{N} \mathrm{II}]$ interference filters. For $I_{\mathrm{H} \alpha}($ line $) \simeq 3 I_{[\mathrm{N} \mathrm{II}}$ (line) we have:

$$
\left(\frac{I_{[\mathrm{N} \mathrm{III}}}{I_{\mathrm{H} \alpha}}\right)_{\text {observed }}=\left(\frac{I_{[\mathrm{N} \mathrm{II}}}{I_{\mathrm{H} \alpha}}\right)_{\mathrm{Line}}+\Delta \text {. }
$$

$\Delta=\left(\varepsilon^{\prime}-\varepsilon / 3\right) / I_{\mathrm{H} \alpha}$ may be neglected.

\section{Results and Comments}

Figure 3 gives the $[\mathrm{N}$ II] $/ \mathrm{H} \alpha$ variation towards the bright rim for 4 selected directions. This ratio first increases smoothly, then remains practically constant before the bright rim is reached. Its maximum value does not occur in the bright rim itself but in a narrow zone situated between the rim and the dark matter.

It is very difficult to interpret the observed [N II]/H $\alpha$ ratio (Mein, 1968; Baudel, 1970) for these lines do not originate from the same region located along the line of sight. Fortunately Figure 1 shows that there is no noticeable morphological difference between the $\mathrm{H} \alpha$ and $[\mathrm{H} \mathrm{II}]$ images. It is reasonable to assume that the $[\mathrm{N} \mathrm{II]} \mathrm{and} \mathrm{H} \alpha$ lines originate roughly from the same region. With this assumption we have the following formula (Louise, 1969);

$$
\frac{[\mathrm{N} \mathrm{II}]}{\mathrm{H} \alpha}=\frac{10^{4}}{6.2} \cdot T_{e}^{\frac{1}{2}} 10^{-9500 / T_{e}} \frac{N\left(\mathrm{~N}^{+}\right)}{N_{e}} .
$$

$N\left(\mathrm{~N}^{+}\right) / N_{e}$ is the relative abundance of singly ionized nitrogen. It is not certain that this quantity remains constant within a given nebula because of the ionisation structure effect (Hummer and Seaton, 1963).

Consequently the observed $[\mathrm{N} \mathrm{II}] / \mathrm{H} \alpha$ variation must be interpreted as the variation of both $N\left(\mathrm{~N}^{+}\right) / N_{e}$ and $T_{e}$ towards the bright rim. For many theoretical considerations $T_{e}$ must increase towards the bright rim; so, if we assume $N\left(\mathrm{~N}^{+}\right) / N_{e}$ to be constant, we may interpret Figure 3 in terms of variation in $T_{e}$. Doing so, the figure shows that $\mathrm{Te}$ increases smoothly and/or remains constant until the bright rim is reached, and then suddenly increases behind it. There are good reasons to think that 

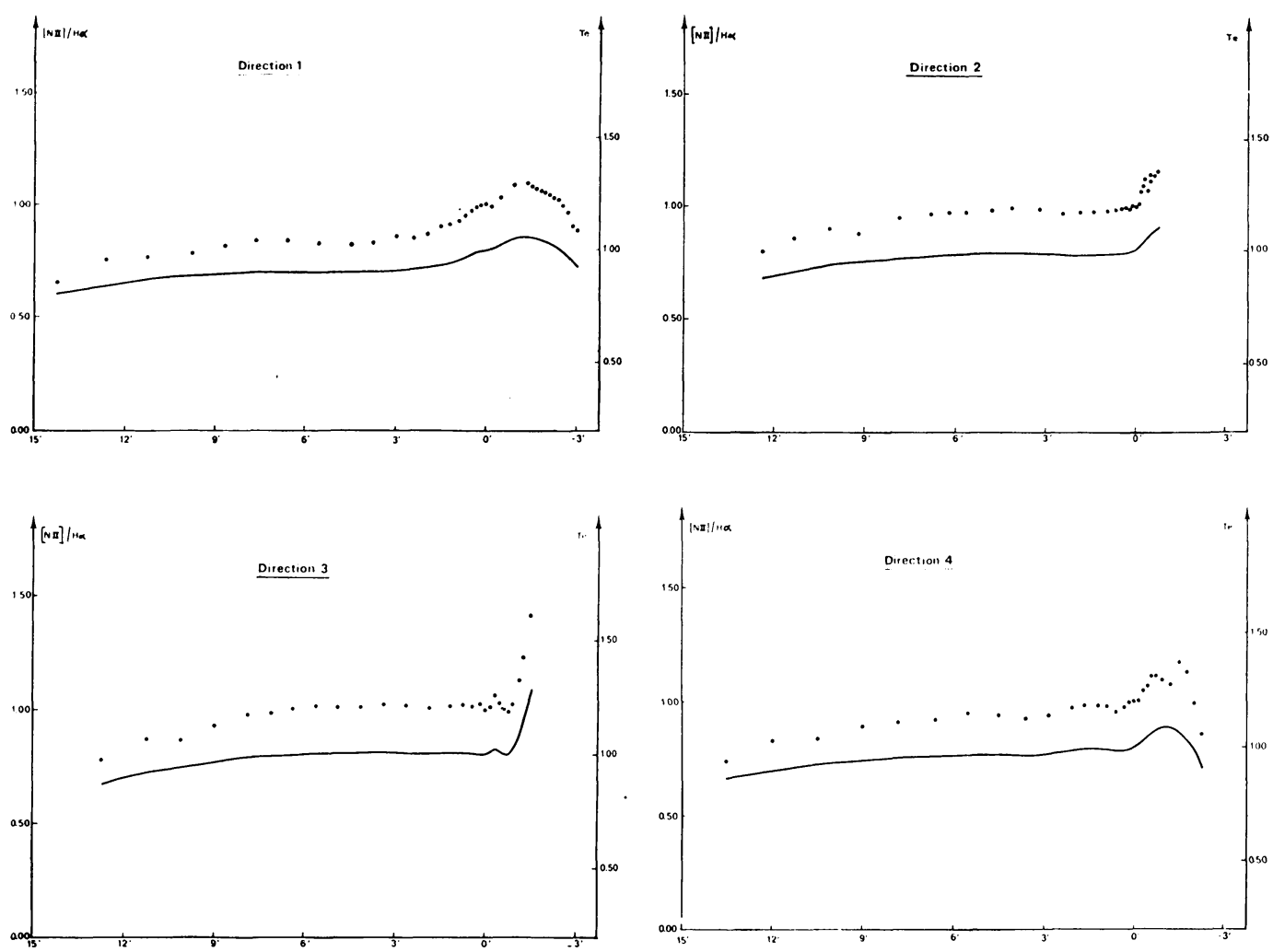

Fig. 3. Values of the observed ratio $[\mathrm{N} I I] / \mathrm{H} \alpha$ (points, left scale) and of the derived electron temperature $T_{e}$ (curve, right scale) along the four scan lines.

this effect is connected with the dissipation of energy of shock waves entering from the $\mathrm{H}$ II zone into the neutral material. The bright rim is certainly a very hot ionization front, but the hottest zone is located between it and the dark matter. This transition zone is very narrow, from $1^{\prime}$ to 1.5 behind the rim.

\section{Conclusion}

The most important study of bright rims is to determine their physical parameters, especially their electron density and temperature. The observation of the $[\mathrm{N} \mathrm{II}] / \mathrm{H} \alpha$ ratio is a very interesting step; but it is not sufficient for a complete study concerning $\mathrm{T}_{\boldsymbol{e}}$ variation towards the bright rim because two fundamental assumptions have to be made:

(a) $\mathrm{H} \alpha$ and [ $\mathrm{N}$ II] originate from the same region;

(b) $N\left(\mathrm{~N}^{+}\right) / N_{e}$ remains constant.

These assumptions are not entirely justified by theoretical considerations but they allow us to obtain the first qualitative results about the electron temperature variation towards the bright rim. 
The maximum value of $[\mathrm{N} \mathrm{II}] / \mathrm{H} \alpha$ just behind the rim remains to be interpreted in a more correct manner than is done in this paper.

Much work remains to be done on this problem. Instead of using [N II] and $\mathrm{H} \alpha$ interference filters, we should observe the bright rims with the filters centered on the [O III] lines. This would allow us to determine directly $T_{e}$ without unacceptable assumptions.

\section{References}

Baudel, L.: 1970, Astron. Astrophys. 8, 65.

Courtès, G.: 1960, Ann. Astrophys. 23, 115.

Courtès, G., Louise, R., and Monnet, G.: 1969, Astron. Astrophys. 3, 222.

Hummer, D. J. and Seaton, M. J.: 1963, Monthly Notices Roy. Astron. Soc. 127, 217.

Louise, R.: 1969, Astron. Astrophys. 3, 29.

Louise, R.: 1970, Astron. Astrophys. 5, 35.

Mein, N.: 1968, Ann. Astrophys. 31, 579.

Osterbrock, D. E.: 1957, Astrophys. J. 125, 622.

Pottasch, S. R.: 1958, Bull. Astron. Inst. Neth. 14, 29. 